\title{
Effects of taurine intake on serum lipids in young women
}

\author{
Sadako Matsui ${ }^{1}$, Chizuko Maruyama ${ }^{1}$, Hiroe Arai ${ }^{1}$, Sachie Hashimoto ${ }^{1}$, Tamami \\ Asakusa ${ }^{1}$, Hiroshi Yoshida ${ }^{2}$, Toshitsugu Ishikawa ${ }^{3}$
}

${ }^{1}$ Department of Food and Nutrition, Faculty of Human Sciences and Design, Japan Women's University, 2-8-1, Mejirodai, Bunkyo-ku, Tokyo, 112-8681, Japan; ${ }^{2}$ Department of Laboratory Medicine, The Jikei University School of Medicine, 163-1, Kashiwashita, Kashiwa-shi, Chiba, 277-8567, Japan; ${ }^{3}$ Department of Nutrition and Life Science, Faculty of Applied Bioscience, Kanagawa Institute of Technology, 1030, Shimo-ogino, Atsugi, Kanagawa, 243-0292, Japan

Corresponding author: Sadako Matsui, Department of Food and Nutrition, Faculty of Human Sciences and Design, Japan Women's University, 2-8-1, Mejirodai, Bunkyo-ku, Tokyo, 1128681, Japan

Submission date: April 27, 2015; Acceptance date: May 27, 2015: Publication date: May 29, 2015

\begin{abstract}
Background: Taurine is an abundant amino acid in human cells, promoting ocular and biliary health, which is also used to treat congestive heart failure, hypertension, and hepatitis. Recently, taurine-enriched energy drinks have become popular with young adults, but the effects of taurine on serum lipids in young adults are unknown.
\end{abstract}

Objective: We studied the influence of oral administration of taurine on serum lipid levels in healthy young women.

Methods: Ten healthy young women with a mean body mass index of $20.0 \mathrm{~kg} / \mathrm{m} 2$, apolipoprotein $\mathrm{E}$ (apoE) phenotype 3/3 and normal menstrual cycles participated. Each subject was instructed to orally ingest $1 \mathrm{~g}$ of taurine powder after each meal (3g/day) in addition to their usual diets during one menstrual cycle. Before and at the end of taurine intake, physical measurements and blood collection were performed in the morning after a 12-h fast, and 3-day weighted dietary records were obtained. Concentrations of serum lipids, apolipoproteins, and fatty acids in the serum phospholipid fraction were measured.

Results: The subjects showed good compliance with taurine intake and none reported adverse effects during the experimental period. After taurine intake, concentrations of total cholesterol, low density lipoprotein cholesterol (LDL-C), free cholesterol, and apolipoprotein B (apoB) increased $(\mathrm{p}<0.05)$, while phospholipids tended to increase $(\mathrm{p}=0.051)$. Fatty acids in the serum phospholipid fraction also significantly increased $(\mathrm{p}<0.05)$. However, triglyceride, remnant-like particle cholesterol, remnant-like particle triglyceride, apoE, the apolipoprotein A-1 (apoA1)/apoB ratio and the LDL-C/apoB ratio were unchanged. Furthermore, body weight was significantly increased $(\mathrm{p}<0.01)$, but did not correlate with changes either in serum lipids or 
nutrient intakes.

Conclusion: These results suggest that high taurine intake affects lipoprotein metabolism and increases serum lipids in slightly lean young women.

Key words: Taurine, slightly lean young women, serum lipids, remnant lipoprotein, fatty acids of the serum phospholipid fraction.

\section{INTRODUCTION}

Taurine is considered to be a semi-essential amino acid, and is the most abundant free intracellular amino acid in human cells [1]. Taurine plays an important role as a basal factor for maintaining cellular integrity in the heart, muscle, retina, and the central nervous system [2]. Physiological effects of taurine supplementation have been reported to include lowering blood pressure [3, 4], antioxidant and anti-inflammatory actions [5], and increased bile acid secretion [6].

Numerous reports have demonstrated that taurine supplementation lowers serum lipid levels, particularly in animal models [7-13]. Taurine supplementation was also found to improve insulin sensitivity [14] and hyperlipidemia in obese and overweight men. However, the effects of taurine supplementation on serum lipids have not been defined specifically in humans [15-17].

In the past two decades, the number of lean young women with a body mass index (BMI) below $18.5 \mathrm{~kg} / \mathrm{m} 2$ has increased to more than 20 percent of women in their twenties who live in Japan [18]. This is associated with various reproductive abnormalities, including menstruation disorders, infertility and low birth weight babies [19]. As a result, this excessively low BMI is a clinical problem which requires study and intervention.

Taurine-enriched energy drinks are now being consumed by young adults with the expectation that fatigue will be ameliorated [20,21]. But there is concern that taurine consumption might lead to malnourishment, being potentially related to decreasing serum lipid levels in young women. Therefore, we investigated the effects of taurine intake on blood lipoprotein lipids in healthy young women.

\section{METHODS}

Ten healthy women, all university students with a regular menstrual cycle and the apolipoprotein E (apoE) 3/3 phenotype, were enrolled in the present study. This study was conducted according to the guidelines laid down in the Declaration of Helsinki. However, this study was performed before the institutional review board of Japan Women's University was established. Nonetheless, informed written consent was obtained from all study subjects after the design and objective of the study had been thoroughly explained. Additionally, the present report received ethical approval from the institutional review board of Japan Women's University (No.12) prior to its submission for publication.

Each subject was instructed to orally ingest $1 \mathrm{~g}$ of taurine powder (Taisho Pharmaceutical Co., Ltd, Tokyo, Japan) after each meal (3g/day) throughout one menstrual cycle. Because total cholesterol (TC) and low density lipoprotein cholesterol (LDL-C) were lower during the luteal phase than the follicular phase [22], we set the duration of taurine intake to exclude the 
influences of female hormones. Therefore, these cyclic variations in lipoprotein levels should be considered in the design and interpretation of studies in reproductive-age women and the clinical management of cholesterol in female patients. Before and at the end of taurine intake, physical measurements and blood collection were performed in the morning after a $12-\mathrm{h}$ fast.

TC, high density lipoprotein cholesterol (HDL-C), triglyceride (TG), phospholipid (PL) and free cholesterol (FC) were measured using enzymatic methods (Kyowa Medics Determiner TC, Determiner HDL-C, Determiner TG, Determiner PL and Determiner FC, Tokyo, Japan) at the Otsuka Assay Laboratory (Tokushima, Japan). LDL-C was calculated with Friedewald's formula [TC - HDL-C - (TG/5)]. Remnant-like particle cholesterol (RLP-C) and RLP triglyceride (RLPTG) were measured after RLP immunoseparation using an immunoaffinity gel containing two different immobilized monoclonal antibodies to human apolipoprotein A-1 (apoA-1) and apolipoprotein B-100 at the Japanese Antibody Research Center (Gunma, Japan). ApoA-1, apolipoprotein B (apoB), and apoE were measured by immunonephelometry (Daiichi Kagaku, Apo Auto First, Tokyo, Japan) at the Otsuka Assay Laboratory (Tokushima, Japan). ApoE phenotypes were determined through isoelectric focusing, followed by the immunoblotting method (Phenotyping ApoE, Tokyo, Japan). Lipids were extracted from serum with Folch's method [23], and after isolation of the PL fraction using thin-layer chromatography and then methylation, fatty acids in the PL fraction were analyzed through gas chromatography (GC-12A, Shimadzu, Kyoto, Japan).

The study subjects were asked to maintain their usual lifestyles and dietary habits, and to record their basal body temperature, body weight, health conditions, appetite, presence of diarrhea, amount of exercise, and alcohol intake during the experimental period. Before and at the end of the taurine intake period, 3-day weighted dietary records, which had been kept by the study subjects, were collected after confirmation by interview with a registered dietitian. Nutrient intakes were calculated with WELLNESS/Win software (Top Business System, Okayama, Japan).

The data is expressed as mean \pm standard deviation. Differences in before-and-after mean values were analyzed by the Shapiro-Wilk test. Afterwards, normally distributed variables were analyzed with the paired t-test, and non-normally distributed variables by the Wilcoxon signedrank test. Single linear regression analyses of the changes in body weight and serum parameters before and at the end of taurine intake were conducted employing the Pearson product-moment correlation test on normally distributed variables. Differences were defined as statistically significant at $\mathrm{p}<0.05$. All statistical analyses were performed using SPSS 18.0 software (SPSS Inc., Japan).

\section{RESULTS}

Subject profiles and the intakes of energy and nutrients: The mean age of the study subjects was $21.6 \pm 0.7$ years, and the mean BMI was $20.0 \pm 1.6 \mathrm{~kg} / \mathrm{m} 2$ at baseline, indicating that the study subjects were slightly lean. The menstrual cycle length was $31.9 \pm 5.2$ days (range: 26-45 days), and the mean duration of taurine intake was 32.4 \pm 5.3 days (range: 25-44 days). The taurine intake compliance rate was good at $98.7 \pm 3.2 \%$.

Energy and nutrient intakes, including fatty acids, did not differ significantly before and at the end of the taurine intake period (Table 1). No adverse effects were reported at any time 
during the experimental period.

Table 1: Nutrient intakes based on dietary records

\begin{tabular}{lll}
\hline & Baseline & End of taurine intake \\
\hline Energy (kcal/day) & $1516 \pm 294$ & $1638 \pm 273$ \\
Protein (\% energy) & $15.1 \pm 1.9$ & $14.2 \pm 1.7$ \\
Lipid (\% energy) & $30.0 \pm 4.0$ & $29.5 \pm 2.8$ \\
Carbohydrate (\% energy) & $54.9 \pm 5.1$ & $56.3 \pm 3.5$ \\
Cholesterol (mg/day) & $185 \pm 100$ & $172 \pm 86$ \\
SFA (g/day) & $195.56 \pm 71.57$ & $189.94 \pm 78.47$ \\
MUFA (g/day) & $258.33 \pm 106.10$ & $246.25 \pm 74.35$ \\
n-6 PUFA (g/day) & $143.09 \pm 55.87$ & $145.32 \pm 38.95$ \\
n-3 PUFA (g/day) & $32.39 \pm 11.66$ & $35.84 \pm 13.49$ \\
PUFA (g/day) & $175.47 \pm 63.00$ & $181.15 \pm 50.61$ \\
P/S ratio & $0.9 \pm 0.3$ & $1.1 \pm 0.5$ \\
\hline
\end{tabular}

$\mathrm{n}=10$; Data is presented as means \pm standard deviation.

Dietary records obtained 3 days before and at the end of taurine intake.

SFA: Saturated fatty acid; MUFA: Mono unsaturated fatty acid; $n-6$ PUFA: $n-6$ poly unsaturated fatty acid; $n-3$ PUFA: n-3 poly unsaturated fatty acid; PUFA: Poly unsaturated fatty acid; P/S ratio: Polyunsaturated fatty acid/Saturated fatty acid

Changes in body weight and fasting serum lipids and apolipoprotein concentrations with taurine intake: As shown in Table 2, compared with the baseline values, body weight and BMI were higher at the end of taurine intake $(\mathrm{p}<0.01)$.

Table 2: Changes in body weight, fasting serum lipids and apolipoprotein concentrations, with taurine intake

\begin{tabular}{lll}
\hline & Baseline & End of taurine intake \\
\hline Body weight (kg) & $50.0 \pm 4.6$ & $50.7 \pm 4.6^{* *}$ \\
BMI (kg/m2) & $20.0 \pm 1.6$ & $20.2 \pm 1.7 * *$ \\
TC (mmol/L) & $4.7 \pm 0.5$ & $5.2 \pm 0.8^{*}$ \\
LDL-C (mmol/L) & $2.7 \pm 0.5$ & $3.1 \pm 0.7 *$ \\
HDL-C (mmol/L) & $1.7 \pm 0.4$ & $1.7 \pm 0.3$ \\
LDL-C/HDL-C & $1.7 \pm 0.6$ & $1.9 \pm 0.6 *$ \\
FC (mmol/L) & $1.2 \pm 0.1$ & $1.3 \pm 0.2 *$ \\
RLP-C (mmol/L) & $0.01 \pm 0.02$ & $0.01 \pm 0.01$ \\
TG (mmol/L) & $0.7 \pm 0.2$ & $0.8 \pm 0.3$ \\
RLP-TG (mmol/L) & $0.06 \pm 0.05$ & $0.09 \pm 0.06$ \\
TG/HDL-C & $0.4 \pm 0.2$ & $0.5 \pm 0.2$ \\
PL (mmol/L) & $2.5 \pm 0.3$ & $2.8 \pm 0.3$ \\
apo A-I (g/L) & $1.4 \pm 0.2$ & $1.4 \pm 0.2$ \\
apo B (g/L) & $0.7 \pm 0.1$ & $0.8 \pm 0.2 \#$ \\
apo E (g/L) & $0.0 \pm 0.0$ & $0.0 \pm 0.0$ \\
LDL-C/apoB & $4.1 \pm 0.5$ & $4.2 \pm 0.4$ \\
apoB/apoA-1 & $0.5 \pm 0.2$ & $0.5 \pm 0.1$ \\
\hline
\end{tabular}

$\mathrm{n}=10$; Data is presented as means \pm standard deviation. 
Statistical analysis was performed using paired t-test $(* * \mathrm{p}<0.01, * \mathrm{p}<0.05$ vs baseline) and Wilcoxon signedrank test (\# $\mathrm{p}<0.05$ vs baseline).

BMI: Body mass index; TC: Total cholesterol; LDL-C: Low density lipoprotein cholesterol; HDL-C: High density lipoprotein cholesterol; FC: free cholesterol; RLP-C: Remnant-like particle cholesterol; TG: Triglyceride; RLP-TG: Remnant-like particle triglyceride; PL: Phospholipid; apoA-1: apolipoprotein A-1; apoB: apolipoprotein B; apoE: apolipoprotein E

In all study subjects, fasting serum lipid levels were within normal ranges at baseline. Taurine intake was associated with significant increases in TC from $4.7 \pm 0.5 \mathrm{mmol} / \mathrm{L}$ to $5.2 \pm 0.8$ $\mathrm{mmol} / \mathrm{L}(\mathrm{p}<0.05)$ and in LDL-C from $2.7 \pm 0.5 \mathrm{mmol} / \mathrm{L}$ to $3.1 \pm 0.7 \mathrm{mmol} / \mathrm{L}(\mathrm{p}<0.05)$. Compared to the baseline, FC was significantly increased $(\mathrm{p}<0.05)$ and PL tended to increase at the end of taurine intake ( $\mathrm{p}=0.051$ ). There were no differences in HDL-C, TG, RLP-C or RLPTG between the baseline and the end of taurine intake. The TG/HDL-C ratio was unchanged. As to serum apolipoprotein concentrations, apoB increased from $0.7 \pm 0.1 \mathrm{~g} / \mathrm{L}$ at the baseline to 0.8 $\pm 0.2 \mathrm{~g} / \mathrm{L}$ at the end of taurine intake ( $<<0.05$ ). ApoA-I, apoE, and the apoB/apoA-I ratio did not differ between the baseline and the end of taurine intake.

Changes in the fatty acid composition of the serum PL fraction: Table 3 shows that, compared with the baseline, all other fatty acids except for C14:0 in the serum PL fraction increased about 1.5 fold in saturated fatty acids, 1.7 fold in C18:1, C18:2, and C 20:5, at the end of taurine intake.

Table 3: Changes in fatty acid composition of serum phospholipid fraction with taurine intake ( $\mathrm{mg} / \mathrm{dl})$

\begin{tabular}{lcl}
\hline Fatty acid & Baseline & End of taurine intake \\
\hline C14:0 & $0.3 \pm 0.1$ & $0.3 \pm 0.1$ \\
C16:0 & $18.5 \pm 4.2$ & $28.4 \pm 10.6 *$ \\
C16:1 & $0.3 \pm 0.2$ & $0.5 \pm 0.2 *$ \\
C18:0 & $9.2 \pm 2.4$ & $14.1 \pm 4.1 * *$ \\
C18:1 & $5.0 \pm 1.4$ & $8.7 \pm 2.6 * *$ \\
C18:2 & $13.6 \pm 3.3$ & $23.0 \pm 7.0 * *$ \\
C18:3 & $0.1 \pm 0.0$ & $0.2 \pm 0.1 *$ \\
C20:4 & $4.8 \pm 1.0$ & $7.3 \pm 1.9 * *$ \\
C20:5 & $1.2 \pm 0.6$ & $2.1 \pm 1.0 *$ \\
C22:0 & $0.6 \pm 0.2$ & $0.9 \pm 0.3 *$ \\
C22:5 & $0.5 \pm 0.1$ & $0.9 \pm 0.3 * *$ \\
C22:6 & $4.0 \pm 0.8$ & $6.2 \pm 1.9 * *$ \\
C24:1 & $1.0 \pm 0.4$ & $1.6 \pm 0.5 * *$ \\
others & $4.0 \pm 1.2$ & $6.7 \pm 1.8 * *$ \\
S & $28.5 \pm 6.9$ & $43.7 \pm 10.1 * *$ \\
M & $6.3 \pm 1.9$ & $10.8 \pm 3.1 * *$ \\
P & $24.1 \pm 4.8$ & $39.6 \pm 10.2 * *$ \\
P/S & $0.9 \pm 0.1$ & $0.9 \pm 0.2$ \\
\hline
\end{tabular}

$\mathrm{n}=10$; Data is presented as means \pm standard deviation.

Statistical analysis was performed using paired t-test $\left(* * \mathrm{p}<0.01,{ }^{*} \mathrm{p}<0.05\right.$ vs baseline) and Wilcoxon signed-rank test (\# $\mathrm{p}<0.05$ vs baseline).

S: Saturated fatty acids (total of C14:0, C16:0, C18:0, C22:0); M: Monounsaturated fatty acids (total of C16:1, C18:1, C24:1); P: Polyunsaturated fatty acids (total of C18:2, C18:3, C20:4, C20:5, C22:6) 
P/S: Polyunsaturated fatty acid/Saturated fatty acid

Correlations among changes before and at the end of taurine intake in body weight, serum lipids, apolipoproteins and fatty acid concentrations in the PL: LDL-C change demonstrated a strong positive correlation with change in the apoB concentration $(\mathrm{p}=0.002)$ (Table 4), but not with change in body weight or those in serum PL fatty acid concentrations (data not shown).

Table 4: Correlation coefficients for the degree of change in body weight and serum parameters

\begin{tabular}{|c|c|c|c|c|c|}
\hline & \multirow{2}{*}{$\begin{array}{l}\Delta \mathrm{LDL}-\mathrm{C} \\
(\mathrm{mmol} / \mathrm{L})\end{array}$} & \multirow{2}{*}{$\Delta \operatorname{apoB}(\mathrm{g} / \mathrm{L})$} & \multicolumn{3}{|c|}{ Fatty acids in the PL fraction $(\mathrm{mg} / \mathrm{dl})$} \\
\hline & & & $\Delta \mathrm{S}$ & $\Delta \mathrm{M}$ & $\Delta \mathrm{P}$ \\
\hline$\Delta \mathrm{BW}(\mathrm{kg})$ & 0.325 & 0.517 & -0.123 & -0.191 & -0.062 \\
\hline$\Delta \mathrm{LDL}-\mathrm{C}(\mathrm{mmol} / \mathrm{L})$ & - & $0.858 * *$ & -0.173 & -0.273 & -0.017 \\
\hline$\Delta \operatorname{apoB}(g / L)$ & - & - & -0.232 & 0.104 & 0.105 \\
\hline
\end{tabular}

$\mathrm{n}=10$; Data are presented as Pearson's simple correlation coefficients for the degree of change before and at the end of taurine intake $(* * \mathrm{p}<0.01)$.

BW: Body weight; LDL-C: Low density lipoprotein cholesterol; apoB: apolipoprotein B; PL: Phospholipid; S: Saturated fatty acids (total of C14:0, C16:0, C18:0, C22:0); M: Monounsaturated fatty acids (total of C16:1, C18:1, C24:1); P: Polyunsaturated fatty acids (total of C18:2, C18:3, C20:4, C20:5, C22:6)

\section{DISCUSSION}

In the present study, taurine intake ( $3 \mathrm{~g} /$ day) for a single menstrual cycle increased body weight, LDL-C, FC, apoB and PL fatty acid concentrations in slightly lean young women. Well-known factors that elevate serum cholesterol include increased body weight, increased energy intake, decreased energy expenditure, female hormones, and certain disorders affecting lipid metabolism. We observed no correlations among these lifestyle-related factors in terms of LDLC change in this study.

A previous report revealed that in menstruating women, TC is higher in the follicular by $0.29 \mathrm{mmol} / \mathrm{L}$ on average than in the luteal phase of the menstrual cycle [22]. To exclude the influences of female hormones, we set the duration of taurine intake throughout a single menstrual cycle in each subject. Thus, the LDL-C increase with taurine supplementation is more likely to be independent of the above-described factors.

In a previous study investigating the influences of taurine administration on serum lipids in humans, Zang et al found that taurine supplementation decreased TG and the atherogenic index in overweight and obese adults [12]. However, Mizushima et al reported that $6 \mathrm{~g} /$ day taurine supplementation for 3 weeks induced significant increases in VLDL-C and TG with high fat, high cholesterol, and low polyunsaturated fatty acid / saturated fatty acid (P/S) ratio diets in healthy men [16]. Tanno et al indicated that $3.2 \mathrm{~g} /$ day taurine supplementation on a high cholesterol diet for 2 weeks increased serum LDL-C and biliary cholesterol levels in healthy 
young men [17]. The present study's results in slightly lean young women are similar to those of previous reports, despite low cholesterol intake and a prolonged administration period.

LDL-C is increased by excessive intake of dietary cholesterol and saturated fatty acids, and is decreased by polyunsaturated fatty acid intake. However, since neither dietary cholesterol intake nor the P/S ratio was changed in the study subjects, the increased LDL-C and related lipid profile changes might be independent of fat intake.

Children with cystic fibrosis, caused by exocrine dysfunction, often have extremely fatty stools, which are associated with digestive and developmental disorders. Taurine administration was reported to reduce fatty stools and increase body weight under these circumstances [24, 25]. Although the digestive function of healthy persons would certainly differ from that of cystic fibrosis patients, the increase in body weight and serum lipids with taurine intake in the present study suggests that taurine might improve fat absorption in lean women.

Additionally, we observed almost all fatty acid levels in the serum PL increased by at least 1.5 fold, especially in C18:1, C18:2, and C 20:5. The serum PL concentration, measured as choline derived from lecithin / lysolecithin / sphingomyelin, also increased but the change did not reach statistical significance. This observation raises the possibility that PL molecules other than lecithin / lysolecithin / sphingomyelin might be increased with a significant elevation of FC. In fact, cholesterol esterification could be poor due to relative increase in PL polyunsaturated fatty acids, which have specificity for lecithin-cholesterol acyltransferase. Although this enzyme is key to cholesterol esterification [26], further studies on taurine intake, as well as mass and activity data for lecithin-cholesterol acyltransferase, are necessary.

We also investigated the influences of taurine intake on remnant lipoprotein levels, but no significant changes were found in either RLP-C or RLP-TG. Though the LDL-C/HDL-C ratio was increased by taurine intake, there was no significant change in the apoB/apoA-1 ratio. Furthermore, the LDL-C/apoB and TG/HDL-C ratios did not change in response to taurine intake. Consequently, these results suggest that taurine intake may not increase small dense LDL, one of the atherogenic lipoproteins, despite the increased LDL-C [27, 28]. Therefore, the increase in the LDL-C/HDL-C ratio with taurine intake in this study would not be associated with atherogenic change in the lipoprotein profile.

The taurine powder ( $3 \mathrm{~g} /$ day) administered is known to be associated with a $<0.5 \%$ incidence of adverse effects, such as nausea and diarrhea, but no study subjects experienced such discomfort in the present study. Thus, the administration of taurine powder was considered safe and otherwise harmless for the study subjects.

However, the present study does have several limitations. First, this was a non-randomized controlled study. As a result, the present data did not clarify the mechanism accounting for the effects of taurine on serum lipids. Second, the study subjects were confined to healthy young women. Third, the study size was modest and the follow-up period was short. Nonetheless, future large-scale and long-term studies should be performed based upon this hypothesisgenerating study. Additionally, effects of taurine intake on malnourished males and elderly subjects should be tested in the future.

\section{CONCLUSION}

These results suggest that high taurine intakes from commercial foods affect lipoprotein 
metabolism and increase serum lipids in slightly lean young women. However, larger studies with longer follow-up and a greater variety of subjects are needed to clarify the effects of taurine intake on lipoprotein metabolism.

Competing interests: The authors have no competing interests to declare.

Author's Contributions: Chizuko Maruyama, $\mathrm{PhD}$ and Toshitsugu Ishikawa, $\mathrm{MD}, \mathrm{PhD}$ designed the study; Sadako Matsui, Hiroe Arai, Sachie Hashimoto and Tamami Asakusa conducted the study and analyzed the data; Sadako Matsui, Chizuko Maruyama, $\mathrm{PhD}$ and Hiroshi Yoshida, MD, PhD drafted and revised the manuscript; all authors read and approved the final version of the manuscript.

\section{Acknowledgements:}

This study was supported by Atsushi Yonemura MD, PhD, Shojiro Sawada MD, PhD, and Kei Nakajima, MD, PhD at the National Defense Medical College. The taurine powder was provided by Taisho Pharmaceutical Co., Ltd.

\section{REFERENCES:}

1. Kim SJ, Gupta RC, Lee HW. Taurine-diabetes interaction: from involvement to protection. Curr Diabetes Rev. 2007, 3: 165-175.

2. Ripps H, Shen W. Taurine: A "very essential" amino acid. Mol Vis. 2012, 18: 26732686.

3. Fujita T, Ando K, Noda H, Ito Y, Sato Y. Effects of increased adrenomedullary activity and taurine in young patients with borderline hypertension. Circulation. 1987, 75: 525532.

4. Liu L, Liu L, Ding Y, Huang Z, He B, Sun S, Zhao G, Zhang H, Miki T, Mizushima S, Ikeda K, Nara Y, Yamori Y. Ethnic and environmental differences in various markers of dietary intake and blood pressure among Chinese Han and three other minority peoples of China: results from the WHO Cardiovascular Disease and Alimentary Comparison (CARDIAC) study. Hypertens Res. 2001, 24: 315-322.

5. Milei J, Ferreria R, Llesuy S, Forcada P, Covarrubias J, Boveris A.. Reduction of reperfusion injury with preoperative rapid intravenous infusion or taurine during myocardial revascularization. Am Heart J. 1992, 123: 339-345.

6. Yamori Y, Murakami S, Ikeda K, Nara Y. Fish and lifestyle-related disease prevention: experimental and epidemiological evidence for anti atherogenic potential of taurine. Clin Exp Phamacol Physiol. 2004, 31(Suppl 2): 20-23.

7. Nakaya Y, Minami A, Harada N, Sakamoto S, Niwa Y, Ohnaka M. Taurine improves insulin sensitivity in the Otsuka Long-Evans Tokushima Fatty rat, a model of spontaneous type 2 diabetes. Am J Clin Nutr. 2000, 71: 54-58.

8. Yokogoshi H, Mochizuki H, Nanami K, Hida Y, Miyachi F, Oda H. Dietary taurine enhances cholesterol degradation and reduces serum and liver cholesterol concentrations in rats fed a high-cholesterol diet. J Nutr. 1999, 129: 1705-1712. 
9. Murakami S, Kondo-Ohta Y, Tomisawa K. Improvement in cholesterol metabolism in mice given chronic treatment of taurine and fed a high-fat diet. Life Sci. 1999, 64: 83-91.

10. Balkan J, Kanbağli O, Hatipoğlu A, Kücük M, Cevikbaş U, Aykaç-Toker G, Uysal M. Improving effect of dietary taurine supplementation on the oxidative stress and lipid levels in the plasma, liver and aorta of rabbits fed on a high-cholesterol diet. Biosci Biotechnol Biochem. 2002, 66: 1755-1788.

11. Murakami S, Kondo Y, Toda Y, Kitajima H, Kameo K, Sakono M, Fukuda N. Effect of taurine on cholesterol metabolism in hamsters: up-regulation of low density lipoprotein (LDL) receptor by taurine. Life Sci. 2002, 70: 2355-2366.

12. Matsushima Y, Sekine T, Kondo Y, Sakurai T, Kameo K, Tachibana M, Murakami S. Effects of taurine on serum cholesterol levels and development of atherosclerosis in spontaneously hyperlipidaemic mice. Clin Exp Phamacol Physiol. 2003, 30: 295-299.

13. Chen W, Matsuda K, Nishimura N, Yokogoshi H. The effect of taurine on cholesterol degradation in mice fed a high-cholesterol diet. Life Sci. 2004, 74: 1889-1898.

14. Xiao C, Giacca A, Lewis GF. Oral taurine but not N-acetylcysteine ameliorates NEFAinduced impairment in insulin sensitivity and beta cell function in obese and overweight, non-diabetic men. Diabetologia. 2008, 51: 139-146.

15. Zhang M, Bi LF, Fang JH, Su XL, Da GL, Kuwamori T, Kagamimori S. Beneficial effects of taurine on serum lipids in overweight or obese non-diabetic subjects. Amino Acids. 2004, 26: 267-271.

16. Mizushima S, Nara Y, Sawamura M, Yamori Y. Effects of oral taurine supplementation on lipids and sympathetic nerve tone. Adv Exp Med Biol. 1996, 403: 615-622.

17. Tanno N, Oikawa S, Koizumi M, Fuji Y, Hori S, Suzuki N, Sakuma E, Kotake H, Namai T, Toyota T. Effect of taurine administration on serum lipid and biliary lipid composition in man. Tohoku J Exp Med. 1989, 159: 91-99.

18. Frederick IO, Williams MA, Sales AE, Martin DP, Killien M. Pre-pregnancy body mass index, gestational weight gain, and other maternal characteristics in relation to infant birth weight. Matern Child Health J. 2008, 12: 557-567.

19. The National Health and Nutrition Survey in Japan, 2012: 35. [http://www.mhlw.go.jp/bunya/kenkou/eiyou/dl/h24-houkoku.pdf]

20. Seifert SM, Schaechter JL, Hershorin ER, Lipshultz SE. Health effects of energy drinks on children, adolescents, and young adults. Pediatrics. 2011, 127: 511-528.

21. Yatabe Y, Miyakawa S, Ohmori H, Mishima H, Adachi T. Effects of taurine administration on exercise. Adv Exp Med Biol. 2009, 643: 245-252.

22. Jones DY, Judd JT, Taylor PR, Campbell WS, Nair PP. Menstrual cycle effect on plasma lipids. Metabolism. 1988, 37: 1-2.

23. Folch J, Less M, Stanley GH. A simple method for the isolation and purification of total lipids from animal tissues. J Biol Chem. 1957, 226: 497-509.

24. Darling PB, Lepage G, Leroy C, Masson P, Roy CC. Effect of taurine supplements on fat absorption in cystic fibrosis. Pediatr Res. 1985, 19: 578-582.

25. Carrasco S, Codoceo R, Prieto G, Lama R, Polanco I. Effect of taurine supplements on growth, fat absorption and bile acid on cystic fibrosis. Acta Univ Carol Med (Praha). 1990, 36: 152-156. 
26. Sgoutas DS. Fatty acid specificity of plasma phosphatidylcholine: cholesterol acyltransferase. Biochemistry. 1972, 11: 293-296.

27. Boizel R, Benhamou PY, Lardy B, Laporte F, Foulon T, Halimi S. Ratio of triglycerides to HDL cholesterol is an indicator of LDL particle size in patients with type 2 diabetes and normal HDL cholesterol levels. Diabetes Care. 2000, 23: 1679-1685.

28. Maruyama C, Imamura K, Teramoto $T$. Assessment of LDL particle size by triglyceride/HDL-cholesterol ratio in non-diabetic, healthy subjects without prominent hyperlipidemia. J Atheroscler Thromb. 2003, 10: 186-191. 